\title{
Death Concerns and Other Adaptive Challenges: The Effects of Coalition-Relevant Challenges on Worldview Defense in the US and Costa Rica
}

\author{
Carlos David Navarrete \\ University of California, Los Angeles
}

\begin{abstract}
A relational approach to the psychology of coalitions suggests that certain stimuli that index adaptive problems for which marshaling coalitional support is a reliably adaptive response should elicit increased support of ingroup ideology. Studies from two cultures produced results consistent with this perspective. In Study 1, Costa Rican participants contemplating coalition-relevant scenarios (i.e. social isolation or the need to enlist the help of others in a cooperative task) increased support of ingroup ideology, but that participants contemplating a mortality-salient prime did not. Study 2 replicated these results in an American sample, and explored the moderating effects of individual variation in interdependence and chronic dangerous world beliefs on normative bias. These results suggest that the determining factor cross-culturally in the elicitation of worldview defense may not be mortality concerns per se, but rather the need for coalitional support.
\end{abstract}

KEYWORDS coalitional psychology, Costa Rica, interdependence, intergroup bias, terror management

ACCORDING to terror management theory (TMT; Greenberg, Pyszczynski, \& Solomon, 1986; Greenberg, Solomon, \& Pyszczynski, 1997; Pyszczynski, Solomon, \& Greenberg, 2002) death anxiety is buffered by belief in one's cultural worldview. Faith in a worldview is said to be important in managing the fear of

\author{
Author's note \\ Address correspondence to C. David \\ Navarrete, Department of Psychology, \\ University of California, Los Angeles, 1285 \\ Franz Hall - Box 951563, Los Angeles, CA \\ 90095-1563, USA \\ [email: cdn@ucla.edu]
}


death as worldviews are thought to provide a sense of purpose and meaning by giving the individual a sense of symbolic immortality through a stable belief system that is larger than the individual and persists after the individual's death. TMT advocates claim that, because the individual's worldview provides protection against death concerns, reminding individuals of the prospect of their own death should increase the need for this anxiety buffer (Greenberg et al., 1986). TMT experiments demonstrate that participants asked to contemplate their own deaths more favorably evaluate targets whose attitudes and values are similar to their own, and become less favorable to those with dissimilar views. TMT theorists claim that these changes reflect an attempt by participants to clarify their cultural worldviews, bolstering their ideologies in order to buffer themselves from the fear of death (Pyszczynski et al., 2002).

While TMT has led to an impressive and creative corpus of research, a number of theoretical difficulties with the framework remain. Because such criticisms have been extensively articulated elsewhere (Boyer, 2001; Buss, 1997; Leary \& Schreindorfer, 1997; Navarrete, Kurzban, Fessler, \& Kirkpatrick, 2004), they will not be reprised here. Holding issues of theory aside, a key question for TMT, and one directly relevant to the data presented in this paper, is of the empirical claim that the effects engendered by mortality-salience are specific and unique to the existential problem of death. TMT proponents have explicitly argued that their experimental results are caused exclusively by the salience of death concerns (Arndt, Greenberg, Solomon, Pyszczynski, \& Simon, 1997; Greenberg, Pyszczynski, Solomon, Simon, \& Breus, 1994), have claimed that such effects are uniquely driven by thoughts of mortality (Greenberg et al., 1997) and asserted that their findings remove TMT from the domain of theories that explain increased cultural affiliation in response to self-relevant threats (Pyszczynski, Greenberg, \& Solomon, 1997).

\section{A relational view of coalitional psychology}

In contrast to TMT, the framework with which I begin is one which is consistent with evolutionary game-theoretic models that emphasize the adaptive utility of conformity to social norms that enhance the efficiency of coordination among self-interested actors (McElreath, Boyd, \& Richerson, 2003), and it also draws from relational models of social cognition that recognize the social foundations of attitudes and beliefs (Asch, 1955; Hardin \& Higgins, 1996; Schaller \& Conway, 1999). I argue that increased support of ingroup ideology engendered by exposure to fitness-relevant threats is a predictable output of a system of adaptive mechanisms that facilitate the formation of social networks, interpersonal attachments, and coalitions (for a fuller discussion see Navarrete et al., 2004). If one of the functions of the internalization of norms is to acquire the social benefits of cooperative action, and if such benefits are particularly important in times of need, then natural selection can be expected to have shaped human psychology such that, when confronted with adaptive challenges that can be remedied through coalitional support, individuals will exhibit a strongly pro-normative orientation in the social cognitions that enhance the maintenance and formation of alliances.

One of the consistent findings within social psychology is that people tend to present themselves in ways they believe will lead to social acceptance and liking by others, and therefore alter the contents of their social representations in response to such relational goals (Asch, 1955; Baumeister \& Leary, 1995; Hardin \& Conley, 2001; Milgram, 1974; Schaller \& Conway, 1999). If relationships are affirmed through a perceived achievement of mutual understanding and common values (Hardin \& Conley, 2001), then impression management motives may exert influence on consensually shared mental representations and evaluative assessments of others (Schaller \& Conway, 1999). This should be particularly so in times of need, threat, or other self-relevant adaptive challenges as the mental system that generates 
the mental representations necessary to facilitate alliances should be particularly active in such situations.

Since social relationships are particularly important in times of social conflict or need (Baumeister \& Leary, 1995; Tooby \& Cosmides, 1996), exposure to particular types of stimuli that index such situations, including but not limited to those concerning death, should lead to increases in pro-normative attitudes toward one's relevant reference groups (what TMT theorists term 'worldview defense'). Hence, whereas TMT theorists claim that the aforementioned changes in cognitive states influencing ideology defense are exclusively the result of exposure to death-related stimuli (Arndt et al., 1997; Greenberg, Simon, Harman-Jones, Solomon, \& Lyon, 1995, 1997), the relational view of coalitional psychology presented here predicts that a range of stimuli indexing adaptive challenges should have this effect as well. More specifically, it predicts that stimuli eliciting a normative bias will concern or index situations that pose adaptive challenges for the individual, and are most effectively addressed using the support of allies.

\section{Background}

In support of the claim that ideology-defense effects occur specifically and exclusively because individuals are motivated to buffer themselves from death anxiety, TMT researchers have gone to considerable length to demonstrate that these 'mortality-salience effects' do not emerge from generalized value accessibility, negative affect, or worrisome thoughts, and that aversive thoughts unrelated to death (such as imagining failing an exam or being forced to engage in public speaking) do not engender effects parallel to those elicited by mortality-salience (reviewed in Greenberg et al., 1997). They interpret these findings as supporting the notion that mortality salience effects are 'outside the purview of other theories that might suggest that self-relevant threats would enhance intergroup bias and adherence to cultural values' (Greenberg et al., 1994, p. 635). However, from a coalitional psy- chology perspective, rather than speaking to the uniqueness of mortality-salience in bolstering and clarifying one's commitment to ingroup ideology, these results simply underline the need to view contemporary experiences with an eye toward the environment in which the human mind evolved. From this perspective, the non-death primes previously used in TMT research such as giving a speech or failing an exam should not be expected to provoke the same shifts in normative cognitions since they do not evoke fitness-relevant challenges in which coalitions could conceivably be a part of an adaptive solution to the problem.

In evaluating the evidence for a relational approach to coalitional psychology, Navarrete et al. (2004) employed a research paradigm typical of TMT experiments. However, in addition to the mortality-salience manipulation, other conditions contained priming stimuli designed to induce participants to think about scenarios unrelated to death but relevant to the need for social assistance. Study participants were asked to imagine themselves confronting a particular challenge in which a reasonable solution to the challenge would be the creation and maintenance of important social ties. In addition to the standard mortality-salience prime used in typical TMT research, primes were employed that focused on (a) a threat to one's personal property, and (b) social isolation and separation from important social relationships. ${ }^{1}$ The results showed that study participants exposed to non-death-salient but coalition-relevant themes were found to display greater intergroup bias compared to participants in a control group. A follow-up manipulation check revealed that these effects were not due to mortality concerns, as the primes did not increase death-thought accessibility on a word completion task.

\section{Interdependence}

An important question in cross-cultural research concerns the extent to which individuals in different societies differ with regard to independent versus interdependent construals of the self. This concept is seen by many cross-cultural researchers as the most basic 
understanding of a culture, and a distinctive dimension of cultural variation (Fiske, Kitayama, Markus, \& Nisbett, 1998). An independent self emphasizes values such as selfreliance, individual rights, and self-actualization. Conversely, the interdependent self focuses on sociocentric values such as dependence on others, duty, and conformity to social norms. Rural Latin American cultures are believed to shape individual thought processes toward an orientation of an interdependent self-construal (Avendano Sandoval \& Dias Guerrero, 1992; Triandis, 1995; Triandis, Marin, Lisansky, \& Betancourt, 1984). Central Americans have been shown to exhibit particularly strong collectivist sentiments and interdependent selfidentities. Hofstede (1991) found Costa Rica, Panama, and Guatemala to be the among the most collectivist out of 52 cultures studied.

Since TMT and the coalitional psychology perspective both postulate the existence of panhuman psychological mechanisms, confirmatory empirical evidence should not be derived exclusively from a narrow experimental context and subject pool. Examination of terror management and coalitional psychology approaches thus requires research studies with participants drawn from cultures which vary with regard to both the centrality of death as a source of anxiety $^{2}$ and the extent to which interdependence and reliance on others to help meet one's life challenges have been culturally elaborated. Because the ability to acquire and use cultural information has been an important determinant of fitness throughout the history of our species (Boyd \& Richerson, 1985, 1990), the behavioral and cognitive outputs generated by pan-human psychological mechanisms should be sensitive to the local cultural context. In order to explore how such information impacts the working of the system in question, it was necessary to examine the impact that cultural differences in the interdependent self might have on the expression of normative bias. In addition to these considerations, a population literate enough to understand the concept of an attitudinal survey was sought, but one in which most people have not been exposed to a university environment.
Finally, given the widespread use of national identification as a dependent measure of ingroup bias in TMT research, expediency concerns required that the studies be conducted with participants having a strong nationalist identity who, like many Americans, derive part of their self-identity as group members of a nation-state. ${ }^{3}$ Given these considerations, rural Costa Rica provided an optimal setting to test both terror management and a relational theory of coalitional psychology while exploring between-culture differences in ethnocentric evaluations of similar and dissimilar others.

The initial studies found that social isolationsalience produced greater pro-normative cognitions than did mortality-salience among Costa Rican participants, but that the opposite was true among American undergraduates (Navarrete et al., 2004). In fact, mortalitysalience produced no significant main effect on normative bias in the Costa Rican samples. This led to the hypothesis that cultural differences in the conceptualization and importance of social relationships might be the cause of these differences. Since rural Costa Ricans value interconnectedness and view their ability to meet life challenges as dependent on their relationships with others, the notion of complete social isolation may be internally assessed as a self-relevant fitness challenge more dire than that assessed by the more individualist-oriented UCLA undergraduates. In short, the motivation to form the necessary bonds with relevant ingroup members in such a situation may be greater in those who feel dependent on others to meet fitness challenges than in those who do not. If correct, this suggests that, within a single culture, interindividual variation in the perceived importance of others in meeting life challenges should correspond with the extremity of pro-normative sentiments associated with that end that are exhibited under conditions of threat or challenge. A follow-up study in Costa Rica produced evidence supporting this prediction (Navarrete et al., 2004, Study 4). In that study, it was found that adherence to collectivist values positively predicted normative bias as a function of exposure to coalition relevant primes, and that increases in normative bias after the manipulation was 
primarily driven by participants with high interdependent self-construals.

\section{The present research}

The initial studies showed the effectiveness of using coalition-relevant primes with aversive themes in increasing intergroup bias, demonstrating that such effects are not limited to those engendered by mortality concerns. In the present research, this enterprise is extended by employing a prime neutral in its affective content, but that speaks directly to the intent and nature of the postulated psychological mechanisms responsible for engendering ideology-defense effects. In the following experiments, 'worldview-defense effects' were sought by using a prime that unambiguously asks participants to contemplate a situation in which they would need to enlist the help of others to solve a cooperative task. If, when faced with adaptive challenges requiring social support, individuals become more normative in their mental representations and evaluative assessments of others in order to facilitate the formation of important social bonds, research participants induced to contemplate enlisting coalitional support to meet a specific adaptive problem should display greater bias toward ingroup ideology when compared to participants contemplating a neutral theme. Therefore, in addition to replicating some of the results that were found in previous experiments using primes with aversive but non-deathrelated coalition-relevant content, the present studies call attention to the utilization and effectiveness of a novel prime that is directly relevant to the workings of the postulated psychological mechanisms that undergird coalitional psychology-a prime that requires participants to contemplate the need to enlist the aid of others to build a home.

The effects of individual and between-culture differences in independent versus interdependent self-construals on normative bias are also explored in the present research. Previous research found that, when induced to contemplate social isolation, Costa Rican participants who scored high on a measure of adherence to collectivist values displayed increased pronormative bias, but those who scored low on this measure did not. Here those findings are replicated in a North American sample, while the effects of individual differences in 'dangerous world' beliefs on pro-normative bias are also explored.

\section{Study 1}

As described above, previous work reveals that Costa Rican participants induced to contemplate social isolation respond with greater defense of ingroup ideology, but that contemplation of death either fails to produce the same effects or only marginally affects normative bias when interacting with moderating variables. One purpose of Study 1 was to explore to what extent both the positive (social isolation) and the null (death salience) results are replicable (or not) among rural Costa Ricans. Study 1 also attempted to demonstrate that increases in normative bias can be engendered by contemplation of the need to recruit coalitional aid to build a home.

\section{Method}

Participants Participants were 120 Costa Rican citizens ( 39 men and 81 women) living in the rural community of Cariari, in the Limon Province of the Republic of Costa Rica-a region with a primarily agricultural economy in which bananas are the primary export (Beisanz, Beisanz, \& Beisanz, 1999). Participants ranged in age from 16 to 65 (mean age: $29.9, S D=11.2$ ) with $60 \%$ of participants over the age of 24 . Education ranged from 0 to 15 years (mean 7.9, $S D=2.5$ ) with the modal education level at the completion of primary school (6 years). ${ }^{4}$ Participants were recruited door-to-door in their homes to participate in a survey on personality and social attitudes. Because the literacy levels of participants varied greatly, surveys were conducted in a structured oral interview format where participants were not required to read research materials (Bernard, 1995; Crano \& Brewer, 2002). All interviews were conducted in informal Spanish by a Costa Rican research assistant who was blind to the hypotheses tested. 
Procedure Each item was read aloud to participants, after which they were asked whether they agreed, disagreed, or were indifferent to the statement. All items from questionnaires were scored on a 3-point scale $(1=$ disagree, $2=$ neither agree nor disagree, $3=$ agree).$^{5}$ The interview began with a scale gauging ethnocentrism, loosely based on the Manitoba Ethnocentrism Scale (Altemeyer, 1996), employed primarily to familiarize participants with the format. Participants were then randomly assigned to a control or an experimental condition. Mortality-salience was manipulated using a prime frequently employed in terror management research (Pyszczynski et al., 2002). Participants were asked to describe the emotions that the thought of their death arouses in them, and to describe what will happen as they physically die. The home building-salience condition entailed a similar open-ended probe, but asked participants to imagine that they had recently purchased a plot of land but, because of limited personal resources, needed the help of family and friends to construct a house. They were then asked to (a) describe the emotions that the prospect of having to ask for the help of family and friends aroused in them, and (b) describe what they would do if confronted with the necessity to enlist the help of others in order to solve such a cooperative task. Participants in the social isolation-salience condition were asked to imagine themselves completely isolated from family and friends, and to describe what they would do if they were completely alone. Participants in the control condition were not primed.

Following the manipulation, the Positive and Negative Affect Schedule, Short Form (PANAS; Watson, Clark, \& Tellegen, 1988), was administered as a delay and distraction before administering the primary dependent measures. ${ }^{6}$ Participants were then read two fictitious essays allegedly written by immigrant authors from another Central American country; the essays concerned the authors' experiences in Costa Rica. One author praised Costa Rican people and their values, while the other criticized Costa Rica. The content of the essays was virtually identical to that of essays used in TMT research. Following each essay, participants were prompted with questions inspired by the Interpersonal Judgment Scale (IJS; Byrne, 1971). Participants were asked to what extent they thought the author was likable (e.g. author was not likable, author was of average likability, author was likable, etc.), intelligent, knowledgeable, moral, mentally well-adjusted, and truthful and the extent to which the participant would want to work with the author. The interview concluded with a series of demographic questions.

\section{Results and discussion}

Normative bias was operationalized as a bias toward Costa Rican ideology evidenced by the difference between participants' IJS ratings of the pro- and anti-Costa Rican authors. The IJS scales for the pro- and anti-Costa Rican authors had Cronbach's alpha reliability ratings of .83 and .87 , respectively. The dependent measure of pro-Costa Rican ideology was calculated by subtracting the mean rating of the anti-Costa Rican target from the mean rating of the proCosta Rican target.

In assessing predictions, a one-way analysis of variance (ANOVA) was conducted with proCosta Rican bias as the dependent variable and condition as the categorical predictor. The analysis revealed a marginally significant main effect for condition $(F(3,116)=2.50, p=.06)$. Planned comparisons regarding the predicted effect of each experimental condition versus the control revealed that contemplation of home building or social isolation-salience led to increased pro-Costa Rican bias, but that contemplation of death did not (Table 1). There were no significant differences among experimental conditions. ${ }^{7}$

These results support the prediction that Costa Ricans induced to contemplate selfrelevant but death-irrelevant adaptive challenges that can be efficiently addressed through enlisting coalitional aid would increase adherence to and support of the ideology of the ingroup. Participants asked to describe their thoughts and what they would do in the event that they were completely socially isolated or 
Table 1. Means and standard deviations of pro-Costa Rican bias by condition (Study 1)

\begin{tabular}{lccccc}
\hline & \multicolumn{5}{c}{ Pro-Costa Rican bias } \\
\cline { 2 - 6 } Condition & Mean & $S D$ & $N$ & $F$-value & Effect size \\
\hline Control & 0.23 & 0.63 & 30 & - & - \\
Mortality & 0.45 & 0.74 & 30 & 1.42 & .30 \\
Home building & 0.67 & 0.67 & 30 & $5.90^{*}$ & .62 \\
Social isolation & 0.63 & 0.72 & 30 & $5.03^{*}$ & .57 \\
\hline
\end{tabular}

$* p<.05$.

Notes: Pro-Costa Rican bias scores ranged from -.88 to 2, with higher scores reflecting greater pro-Costa

Rican bias. F-values reflect contrasts versus the control. Effect sizes calculated as Cohen's D.

needed support from significant others in order to build a home showed an increase in normative Costa Rican bias when compared to a control group. Consistent with previous research (Navarrete et al., 2004), Costa Rican participants contemplating their own death showed no significant increase in normative bias compared to the control.

These findings are consistent with the notion that differences exist between cultures in the extent to which particular classes of solutions to adaptive challenges are culturally elaborated. Various aspects of the relational needs observed by people in every society may be cognized differently across cultures, causing particular scenarios to be markedly aversive in a given society. Social isolation may be perceived as a more significant threat in Costa Rica, a society that emphasizes interdependent responses to life challenges (Beisanz et al., 1999), while death fears may not be particularly salient to individuals in such a society where religious beliefs (e.g. Day of the Dead celebrations) and fatalistic attitudes make exposure to lethal themes less problematic than for people living in societies which emphasize secular life and control over one's fate.

\section{Study 2}

Study 1 found that Costa Rican participants induced to contemplate social isolation or coalitional need (scenarios unrelated to mortality concerns) became more biased toward ingroup ideology. In the second study, a replication of these effects was sought among urban university undergraduates in Los Angeles, California. Study 2 attempted a replication of the above-described interaction effects between interdependence and experimental prime found in Costa Rica (Navarrete et al., 2004, Study 4) among a North American undergraduate population. Likewise, data comparable to that previously collected in Costa Rica was sought in order to examine the contribution of cultural differences in interdependent self-construal to the observed cultural differences in intergroup bias toward the ideology of the ingroup.

\section{Chronic beliefs about danger}

In Study 2, the possibility that chronic beliefs about danger and adaptive challenge may interact in the activation of coalitional mechanisms was explored. This exploration was conducted on the assumption that one's belief in the level of danger in the environment is associated with one's self-assessment of vulnerability to harm (Schaller, Park, \& Mueller, 2003). Because individuals who perceive the world to be a dangerous place can be expected to make particularly strident efforts to protect themselves, when confronted with potentially threatening situations in which social support is valuable, such individuals ought to evince marked increases in the pro-normative social cognitions that facilitate the recruitment of allies. Therefore, the procedure included the administration of a scale gauging chronic dangerous world beliefs before the manipulation as an index of one's self-perceived vulnerability to harm, predicting positive interactions for 
experimental conditions with scores on chronic beliefs in a dangerous world.

\section{Method}

Participants UCLA undergraduates were recruited to participate for a payment of US $\$ 5.00$ in a study described as an anonymous survey of personality, social attitudes, and interpersonal evaluation. Participation in the study was restricted to US-born citizens in order to increase the effectiveness of the dependent measure of pro-American bias by eliminating those for whom the US was not an ingroup. Initially, 126 participants volunteered for the study. After removing responses from two participants who failed to complete all items on the questionnaires, data were analyzed for 78 women and 46 men. Participants ranged in age from 18 to 40 years (mean age $=20.5, S D=2.6$ ) with $95 \%$ of participants under the age of 24 . Years of education ranged from 12 to 15 (mean $=13.6, S D=1.3)$.

Materials and procedure Participants were randomly assigned to one of four priming conditions: mortality-salience, home constructionsalience, social isolation-salience, and a control group. Upon arrival at the lab, participants were given a packet containing a series of questionnaires that included one of the primes. Participants were instructed by research assistants to complete the packet of questionnaires at their own pace, taking care to complete them in the order in which they were presented in the packet. The survey packet was separated into three sections of questionnaires. Participants began with an initial battery of filler questionnaires, described as a survey of social attitudes. Embedded among these questionnaires was the 23-item ESTCOL collectivism scale (Realo, Allik, \& Vadi, 1997) and the six-item Belief in a Dangerous World scale (Altemeyer, 1996). Three novel items were added to the Dangerous World scale to complement its face validity as a measure of chronic beliefs about vulnerability to danger in the world. These items were (1) I often fear for my safety, (2) I often fear for the safety of friends and family, and (3) The world is a dangerous place. Following the initial battery of questionnaires, participants began on the second section of the packet, described as a personality inventory. This section included the respective materials for the four conditions that were randomly assigned to each packet. In a section described as a life-attitudes assessment that reveals significant aspects of one's personality, participants were prompted to complete one of four open-ended questionnaires, constituting the prime for each condition. Participants in the treatment conditions were instructed to contemplate and comment on the content of the primes described in Study 1 (i.e. mortality, home building, or social isolation-salience). Similar to a typical neutral prime used in TMT research, participants in the control condition were asked to describe their emotional and physical state as they imagined watching their favorite television program. Following the manipulation, participants completed the state PANAS-X (Watson \& Clark, 1992) as a delay and distraction (Arndt et al., 1997; Greenberg, et al., 1990). Participants then began the third section of the packet, described as an investigation of interpersonal attitudes gauging how Americans react to the impressions of the United States held by people from foreign countries. The section began with two short essays putatively written by two foreign students. One essay presented the experiences and opinions of an author critical of the US and its citizens, the other praised America and American values. Each essay was followed by the IJS (Byrne, 1971) described previously to measure participants' subjective evaluations of the target authors. The questionnaire packet concluded with several demographic items, after which participants were instructed to seal their packet in a large manila envelope and deliver it to the research assistant. Finally, participants were debriefed, thanked, and paid for their participation.

\section{Results and discussion}

As in Study 1 and in TMT research, proAmerican bias was calculated by subtracting the mean IJS rating of the anti-US target from the mean rating of the pro-US target. The IJS 
scales for the pro and anti-US authors had Cronbach's alpha reliability ratings of .78 and .88 , respectively.

In assessing the effects of experimental manipulations on normative bias, a one-way ANOVA was conducted where pro-American bias was the dependent variable and experimental condition was the categorical predictor. The results of the omnibus ANOVA revealed a significant main effect for condition $(F(3,120)=$ $4.13, p<.001)$. Planned comparisons of the predictions for each experimental condition versus the control revealed that contemplating death, home construction, or social isolation-salience themes each led to increased pro-American bias (Table 2). There were no significant differences among experimental conditions.

These results provide clear support for the prediction that confronting self-relevant adaptive challenges, the solution to which includes the support of coalitional allies, increases the pro-normative social representations necessary for creating or reaffirming social bonds. These effects are not exclusive to themes that invoke mortality concerns, as increases in normative bias were found not only for participants induced to contemplate their own death, but also for participants contemplating social isolation or the need for aid in building a home. Particularly with regard to the latter, these findings provide a cross-cultural replication of the novel results of Study 1. There was also a replication of the finding that, among American undergraduates, mortalitysalience engendered greater normative bias than did social isolation-salience, although the differences were not significant (Navarrete et al., 2004). This is consistent with the notion that, in a culture that emphasizes individualism over interdependence, the threat of social isolation may not be experienced as a fitness challenge of the same magnitude as a variety of other adaptive problems (Navarrete et al., 2004). Support for this explanation is assessed below using a between-individuals test of the previous finding that normative bias increases in reaction to adaptive challenges primarily among participants possessing relatively interdependent self-construals.

Interdependence In evaluating the prediction that the increase in normative bias after exposure to adaptive challenges would be particularly strident among participants reporting a high degree of interdependent self-construals, a multiple regression was conducted with proAmerican bias as the dependent variable, and with the experimental conditions, collectivism (Realo et al., 1997), and their cross-products as the predictors. ${ }^{8}$ Both the continuous dependent and independent variables were standardized (mean $=0, S D=1)$, and the dichotomous variables for experimental conditions were dummy coded (Aiken \& West, 1991). ${ }^{9}$

The regression revealed that, controlling for the effects of interdependence, exposure to the experimental primes significantly increased pro-American bias when simple main effects for all experimental conditions were assessed at the grand mean $(F(1,116)=13.56, p<.001)$. Differences among experimental conditions were not significant $(F(2,116)=1.26, p=.29)$. A contrast

Table 2. Means and standard deviations of pro-American bias by condition (Study 2)

\begin{tabular}{lccccc}
\hline & \multicolumn{5}{c}{ Pro-American bias } \\
\cline { 2 - 6 } Condition & Mean & $S D$ & $N$ & $F$-value & Effect size \\
\hline Control & 0.16 & 1.09 & 31 & - & - \\
Mortality & 1.02 & 1.34 & 29 & $6.71^{* *}$ & .65 \\
Home building & 1.21 & 1.60 & 37 & $11.37^{* * *}$ & .75 \\
Social isolation & 0.83 & 0.88 & 27 & $4.81^{*}$ & .50 \\
\hline
\end{tabular}

$* p<.05 ; * * p<.01 ; * * * p<.001$.

Notes: Pro-American bias scores ranged from -2.57 to 5.00 , with higher scores reflecting greater pro-American bias. Effect sizes calculated as Cohen's D. 
comparing the collectivism $\times$ experimental condition interaction versus the control slope revealed that pro-American bias increased as a function of collectivism and exposure to the experimental primes $(F(1,116)=3.83, p=.05)$.

On a test of the specific prediction that contemplating death, home construction, and social isolation-salient themes would increase pro-American bias when interdependent selfconstruals were high, but not when such selfconstruals were low, pro-American bias was assessed for each experimental condition at high and low levels of interdependence. When simple effects for normative bias were assessed at 1 standard deviation above the grand mean, simple effects for experimental condition were significantly higher than that of the control $(F(1,116)=14.48, p<.001)$, with no significant differences among experimental conditions $(F(2,116)=1.13, p<.33)$. Planned comparisons of each experimental condition versus the control when interdependence was high each showed that exposure to mortality, home building, and social isolation-salient primes each increased pro-American bias above the level of control participants at the same level of interdependence (Figure 1). Simple effects assessed at low levels of interdependence (1 SD below the mean) revealed no significant differences between experimental condition and control, with no significant differences among experimental conditions (Figure 1).

These data provide solid cross-cultural support for the hypothesis that, within a single culture, individuals who vary in their selfassessment of the importance of interconnectedness should react differentially to fitness threats, since those whose self-assessed high dependence on others to meet life challenges should exhibit more strident pro-normative mental representations that form the cognitive substrates facilitating social support. At a subjective level, the motivation to form the necessary bonds with relevant ingroup members when confronting adaptive challenges may be more critical for those who feel dependent on

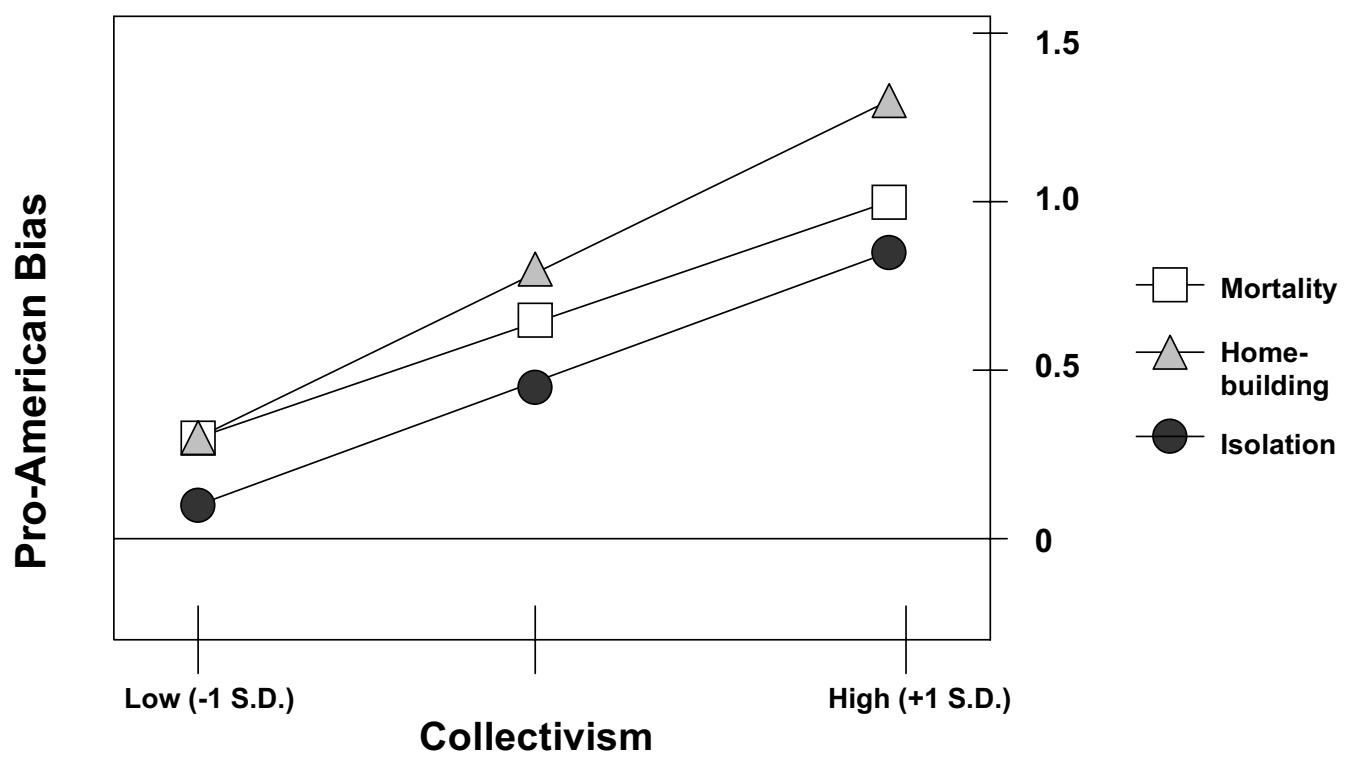

Figure 1. Interaction predicting pro-American bias as a function of collectivism and experimental condition. Symbols represent simple effects for pro-American bias for each experimental condition versus the control when interdependence is high (1 SD above the grand mean), low (1 SD below the mean), and when collectivism is held constant at the grand mean. Effects shown in standardized beta-weights. 
others to meet fitness challenges than for those who do not. This interpretation is consistent with the finding (here and in previous research) that, among UCLA undergraduates, normative bias is greater after exposure to mortality-salient primes than after expsoure to isolation-salience, whereas the reverse pattern is found among the Costa Rican samples. This suggests that observed main effect differences in normative bias between rural Costa Ricans and urban North American undergraduates with regard to the threat of social isolation or death anxiety is attributable to cultural differences in interdependent self-construals between these two populations.

The above account suggests that measurable differences in mean levels of interdependence should be found between both populations. To test this hypothesis data from previous work in Costa Rica were revisited in which interdependence was assessed by administering a Spanish- language version of the collectivism scale used here in Study 2. Participants were 87 Costa Rican citizens (38 men and 49 women; mean age $=33.8$ ) living in the same rural areas in which the data for both Study 1 and previous experimental work were collected (Navarrete et al., 2004; for a full description of methods and descriptive statistics see Navarrete, 2004, chapter 1, Pilot Study 3). A $t$ test was conducted comparing the mean level of interdependence between rural Costa Ricans and the sample of UCLA undergraduates described above. The results revealed that mean interdependence was indeed substantially higher among rural Costa Ricans $(M=3.65, S D=.56)$ than among UCLA undergraduates $(M=2.91, S D=.46)$ $(t=10.47, p<.00001)$.

Chronic dangerous world beliefs In evaluating the secondary hypotheses concerning the effects of chronic beliefs about danger on

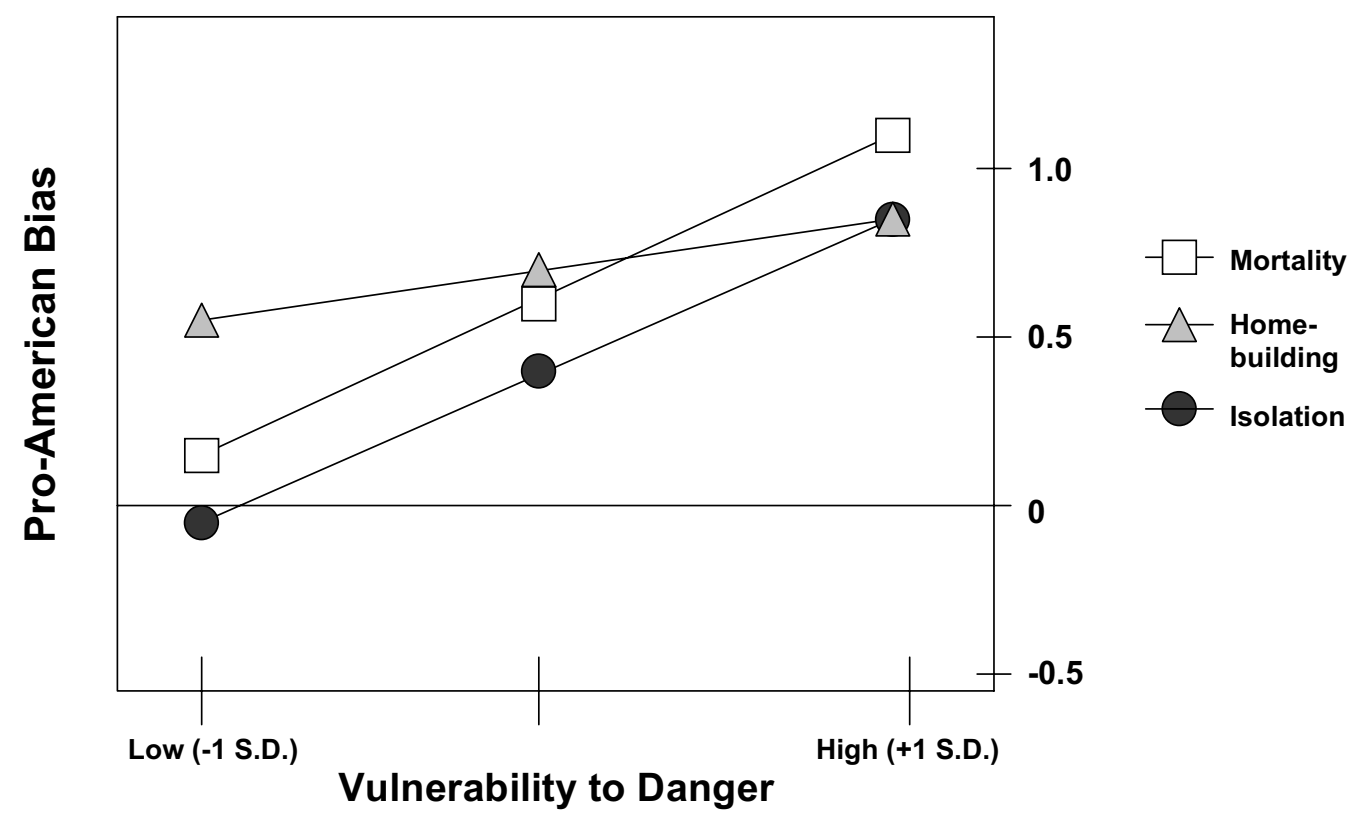

Figure 2. Interaction predicting pro-American bias as a function of chronic dangerous world beliefs and experimental condition. Symbols represent simple effects for pro-American bias for each experimental condition versus the control when vulnerability to danger is high (1 SD above the grand mean), low (1 SD below the mean), and when danger vulnerability is held constant at the grand mean. Effects shown in standardized beta-weights. 
normative bias, a separate regression analysis was conducted with pro-American bias as the dependent variable and experimental manipulations, dangerous world beliefs, ${ }^{10}$ and their interactions as predictors. The dangerous world scale was standardized and experimental conditions were dummy-coded.

The analysis revealed that, controlling for individual differences in dangerous world beliefs, exposure to the experimental primes significantly increased pro-American bias when simple main effects were assessed at the grand mean $(F(1,116)=11.95, p<.001)$. Differences among experimental conditions were not significant. A comparison of the combined dangerous world slopes of the experimental conditions versus the slope of the control revealed a marginal increase in pro-American bias as a function of dangerous world beliefs and exposure to the experimental primes $(F(1$, $116)=3.15, p=.08)$. Differences among the slopes among the experimental conditions were not significant. However, consistent with predictions, the interaction occurred primarily as a function of exposure to the death and isolation-salience primes $(F(1,116)=5.35$, $p<.05)$, as the slope for the home buildingsalience condition was not significant (Figure 2).

On a test our more central prediction that contemplating coalition-relevant themes would increase pro-American bias when chronic beliefs of danger in the world were high, but not when low, simple effects of pro-American bias for the mortality and social isolationsalience conditions were assessed at high and low levels of dangerous world beliefs. When simple effects for normative bias were assessed at $1 S D$ above the grand mean, a contrast of all experimental conditions versus the control were significant $(F(1,116)=12.60, p<.001)$, with no significant differences between experimental conditions. A simple effect assessed at low levels of dangerous world (1SD below the mean) revealed no significant differences between all experimental conditions and control. There were no significant differences among experimental conditions.

The results of Study 2 were consistent with expectations regarding the interaction of dangerous world beliefs and fitness threats in engendering intergroup bias. Participants whose chronic perception of vulnerability to harm was high exhibited more strident bias toward American ideology after contemplating death or isolation than those who rated themselves low on dangerous world beliefs. Also consistent with expectations was the finding that this interaction did not reach significance for participants who contemplated enlisting support to build a home, as it is not obvious that such concerns speak to the need for safety from danger. These results are consistent with the notion that a latent relationship between intergroup bias and one's self-assessed vulnerability to danger can be activated by particular classes of fitness threats through the activation of mechanisms of coalitional psychology.

\section{General discussion}

Taken together, the results of the present experiments provide strong support for the stated hypotheses, and are consistent with the relational theory of coalitional psychology outlined above.

Study 1 demonstrated that rural Costa Rican participants induced to contemplate a scenario involving social isolation became more pronormative toward ingroup ideology. Using the content of a dependent measure of adherence to the normative ideology of the ingroup similar to that typically employed in TMT research, Costa Rican participants who contemplated their own death were found to show no increase in normative bias compared to a control. These results replicated previous findings of two studies (Navarrete et al., 2004, Studies 3 \& 4) that showed no main effect for mortality-salience in engendering adherence toward the ideology of the ingroup among Costa Rican participants. The results of Study 1 also demonstrated the efficacy of a novel prime in producing parallel effects of normative bias, a prime with high face validity for the theory of coalitional psychology in that it induces study participants to contemplate the need for social support to solve a life challenge. Supporting the view that worldview defense effects are not 
uniquely driven by thoughts of mortality but rather reflect the need for coalitional support, participants who contemplated the need to enlist support from their family and friends to build a home increased their support for the ideology of the ingroup.

In Study 2, elements of these results were replicated in a sample of urban, US-born undergraduates at the University of California, Los Angeles. Study 2 revealed that students contemplating social isolation, the need for social support, or their own death showed increased bias toward the normative ideology of the ingroup. These results replicate previous research in that contemplation of social isolation produced smaller effects than did mortality-salience among UCLA undergraduates (although these effects were not significant), and highlight the novel finding that contemplation of the need to recruit aid for a cooperative task increases pro-normative ideology.

These results also contact cultural differences between UCLA participants and Costa Rican participants, as the two populations differ with regard to the relative strength of the impact of social isolation and mortality-salience on normative bias. These population differences in ethnocentric responses are consistent with the notion that ideological reactions to self-relevant adaptive challenges reflect cultural differences in how such challenges are cognized. Various aspects of universal themes addressed in every society may be differentially cognitively elaborated or downplayed in different cultures (Levy, 1973), producing between-population differences in the aversiveness of various scenarios. Specifically, social isolation may be a more significant threat in societies that emphasize collective as opposed to individual responses to challenges, while death fears may not be particularly salient to individuals in societies where religious beliefs and fatalistic attitudes make avoiding potentially lethal themes less troublesome than for people living in complex industrialized nation-states where cultures emphasize secular life, longevity, avoidance of death, and control over one's destiny (Cohen \& Nisbett, 1998; Durkheim, 1951/1897; Kiev, 1976).
Strong evidence for this interpretation came from an analysis of how normative bias is moderated by population and individual differences in construals of the self. Study 2 provided crosscultural support for the notion that increased normative bias after exposure to primes indexing adaptive challenges was driven primarily by participants who scored high on a measure of interpersonal dependence. That is, normative bias increased as a function of both adaptive challenge and interdependence when compared to a control. Evidence supporting the claim that between-population differences in pro-normative responses to social isolation primes are the product of cultural differences in interdependence was supported through a comparison between UCLA undergraduates and rural Costa Rican citizens of the means of a measure of interdependence.

In addition to examining the moderating effects of interdependence, Study 2 also explored the effects on normative bias of individual differences in chronic beliefs in vulnerability to danger. Given that one's self-assessed vulnerability to danger in the environment is predictive of the need to meet dangerous adaptive challenges, it was predicted that the effects of the primes that are relevant to keeping one safe from danger (i.e. social isolation and death salience) should operate differentially as a function of belief in vulnerability to harm. Consistent with expectations, it was found that social isolation and mortalitysalience primes both interacted with dangerous world beliefs in increasing latent normative bias. Normative bias for observed effects was most strident among participants expressing high chronic beliefs in a dangerous world. Consistent with the fact that the opportunity to build a home is not a dangerous situation, no significant interaction with dangerous world beliefs was found for the home buildingsalience condition.

\section{Concluding remarks}

The patterns of these results support the notion that humans possess psychological mechanisms that shape reactions to adaptive challenges. 
These mechanisms adjust behavior as a function of the need to improve one's chances of association and coordination with relevant others, generating output that is shaped by the culture of the group with which one identifies. The evidentiary value of the present findings is strengthened by the fact that many of these results were obtained in a cultural context quite different from a North American university environment, a feature which speaks to the strength of the theory in making predictions about pan-human psychological mechanisms and the manner in which they operate in differing cultural contexts.

This enterprise is not the first to demonstrate that pro-normative ideation rises in response to self-relevant threats that do not engender death-thoughts. Indeed, self-integrity threat manipulations that share theoretical links to uncertainty (McGregor, Zanna, Holmes, \& Spencer, 2001) or body-envelope integrity (Burris \& Rempel, 2004) but not death have been found to increase ideology defense on precisely the same dependent measures used here and in TMT research. The results presented here extend the evidence that worldview-defense effects are not unique to mortality concerns, and can be produced using fitnessrelevant primes without reminding participants of their mortality.

Despite their claims regarding the unique centrality of mortality concerns underlying the defense of ingroup ideology, terror management advocates acknowledge that it is still possible that some yet unspecified category of aversive events is the real culprit responsible for the results of our mortality salience studies' (Greenberg et al., 1994). The relational theory of coalitional theory outlined here specifies that the category of events responsible for such results may be those events that pose adaptive challenges that can conceivably be effectively addressed through coalitional support, of which confronting death is but one. This perspective provides a compelling and coherent account in which to frame the phenomenon of ideological bias impressively documented by TMT research, and one which is amenable to future empirical verification.
To be sure, the results of this program are not entirely inconsistent with terror management theory. Coalitional psychological mechanisms can in fact be operating alongside and complementary to terror management processes, and mortality-salient stimuli may in fact have unique evocative powers on psychological functioning and defensive reactions that operate outside of a coalitional psychological system. However, the relational approach to coalitional psychology outlined here may provide a more parsimonious explanation of the available data to date given that (1) the mechanisms for coalitional computation are likely to be much older phylogenetically than are those designed to assuage existential concerns, as evidenced in the patterns of coalitions and alliances in humans and other animals (De Waal \& Harcourt, 1992; Darwin, 1871), and (2) two theories of worldview defense are not necessary when only one is sufficient. Therefore, a more parsimonious conjecture is that if a unique terror management psychological system exists, it has been 'exapted'11 from an already pre-existing psychological system designed for forming and maintaining alliances. In this regard, the theory of coalitional psychology described here offers an alternative to TMT in explaining the phenomenon of mortality concerns and worldview defense, and also has the potential to restructure social psychological and anthropological knowledge on the general phenomenon of threat and intergroup bias. Further empirical and theoretical work is necessary before such claims can be more forcefully argued.

\section{Notes}

1. The rationale for employing these primes were as follows: the psychological mechanisms that operate to enhance coalitional support should be activated by the prospect of having had one's possessions stolen, for such an event indicates an immediate increase in the need for resources, indexes inadequate social assistance in the protection of one's resources to date, and signals the need for allies in seeking vengeance on thieves, actions that will deter future fitness-reducing transgressions. Likewise, the prospect of complete social isolation as an 
experimental prime was predicted to similarly elicit the mechanisms that enhance coalitional support. An individual's access to any of the benefits of sociality, such as finding mates, protection from predators or conspecifics, access to food and shelter, etc., depends on his or her inclusion in social groups. To the extent that social networks and coalitions aid individuals in procuring these benefits, people should be motivated to take steps to achieve inclusion in relevant social groups. Consistent with our reasoning described above for the theft-salience prime, we posited that the mechanisms of the adaptive system for creating or reaffirming beneficial social bonds in times of social exclusion or marginalization should give rise to the pro-normative, ingroup-affiliative sentiments necessary to obtain needed social support.

2. Many Hispanic authors have noted that death fears are more likely to be expressed openly in many Latin American cultures instead of repressed as they often are in the North (e.g. Fierro, 1980; Delibes, 1966). Death themes have been staples of Hispanic literature for centuries and may have pre-Columbian roots (Siefken, 1993).

3. Costa Rica is a small Central American nation with a population of less than 4 million inhabitants, with about a third of the population living in rural areas. Although still a largely agricultural country, it has achieved a relatively high standard of living and literacy rate compared to other Central American countries. Since 1948, Costa Rica has been an exception in Central America in its lack of political violence, and its citizens are said to exhibit strong pro-nationalist tendencies, believing their country to be unique in its relatively high standards of education, tolerance, and health care in an otherwise turbulent and economically depressed geographic region (Beisanz, Beisanz, \& Beisanz, 1999; Meléndez, 1991).

4. A multivariate regression on demographic variables revealed no significant differences in age, education, or sex ratio across conditions.

5. Pilot studies suggested that Costa Rican participants' inexperience with Likert-type scales made it difficult to gauge their responses using a typical 7- or 9-point scale. Therefore, we simplified the process by limiting the responses to a 3-point scale.

6. Terror management researchers report that a delay and distraction between the death prime and the measurement of worldview defense is necessary for mortality-salience effects to occur, ostensibly because death thoughts are suppressed immediately following mortality-salience induction (Arndt et al., 1997). Without taking a position on this theoretical claim, to provide every opportunity to allow the death prime to work, the PANAS was administered between the manipulation and the measurement of normative bias.

7. Analyses producing $F$-values less than 1 are not reported.

8. The collectivism scale had a Cronbach's alpha reliability rating of .82 . In the regression, the demographic variables of gender and age were initially added. However, these were removed from the model as they did not significantly increase the amount of variance explained by the model.

9. All inferential statistics for the regression analyses were calculated using robust standard errors (Huber, 1981).

10. A factor analysis confirmed that Altemeyer's Belief in a Dangerous World Scale and the three new items formed a single dimension. All combined items loaded above .30 on this dimension. Items combined had a Cronbach's alpha reliability of .84 .

11. This term refers to the term now widely used in evolutionary biology coined by S. J. Gould and E. S. Vrba (1982) to describe the utilization of a structure or feature for a function other than that for which it was developed through natural selection.

\section{Acknowledgments}

This research was supported by a National Science Foundation Postdoctoral Fellowship, a National Science Foundation Dissertation Improvement Grant, UCLA Siff and Fishbaugh Fellowships, and a UCLA Dissertation Year Fellowship.

Thanks to Jim Sidanius, Dan Fessler, Alan Fiske, Nancy Dess, Karthik Panchanathan, Richard McElreath, and two anonymous reviewers for their helpful feedback in an earlier version of this article. I am also grateful to Eric Cubillo Mendoza, Victor Quesada Cruz, and Elier Espinoza Avila for their translation assistance. Lastly, I thank Eric Cubillo Mendoza, Serena Eng, and the Anthropology 197 research assistants for their help in data collection and data entry. 


\section{References}

Aiken, L., \& West, S. (1991). Multiple regression. Newbury Park, CA: Sage.

Altemeyer, B. (1996). The authoritarian specter. Cambridge, MA: Harvard University Press.

Arndt, J., Greenberg, J., Solomon, S., Pyszczynski, T., \& Simon, L. (1997). Suppression, accessibility of death-related thoughts, and cultural worldview defense: Exploring the psychodynamics of terror management. Journal of Personality and Social Psychology, 73, 5-18.

Asch, S. E. (1955). Opinions and social pressure. Scientific American, 193, 31-35.

Avendano Sandoval, R., \& Dias Guerrero, R. (1992). Estudio experimental de la abnegación. Revista Mexicana de Psicología, 9, 15-19.

Baumeister, R. F., \& Leary, M. R. (1995). The need to belong: Desire for interpersonal attachments as a fundamental human motivation. Psychological Bulletin, 117, 497-529.

Beisanz, M. H., Biesanz, R., \& Biesanz, K. (1999). The Ticos: Culture and social change in Costa Rica. London: Lynne Rienner.

Bernard, R. H. (1995). Research methods in anthropology. Qualitative and quantitative approaches (2nd ed.). London: Sage.

Boyd, R., \& Richerson, P. J. (1985). Culture and the evolutionary process. Chicago: University of Chicago Press.

Boyd, R., \& Richerson, P. J. (1990). Culture and cooperation. In J. J. Mansbridge (Ed.), Beyond self-interest (pp. 111-132). Chicago: University of Chicago Press.

Boyer, P. (2001). Religion explained: The evolutionary origins of religious thought. New York: Basic Books.

Burris, C. T., \& Rempel, J. K. (2004). 'It's the end of the world as we know it': Threat and the spatial-symbolic self. Journal of Personality and Social Psychology, 86, 19-42.

Buss, D. M. (1997). Human motivation in evolutionary perspective: Grounding terror management theory. Psychological Inquiry, 8, 22-26.

Byrne, D. (1971). The attraction paradigm. San Diego, CA: Academic Press.

Cohen, D., \& Nisbett, R. E. (1998). Are there differences in fatalism between rural Southerners and Midwesterners? Journal of Applied Social Psychology, 28, 2181-2195.

Crano, W. D., \& Brewer, M. B. (2002). Principles and methods of social research (2nd ed.). Mahwah, NJ: Erlbaum.

Darwin, C. (1871). The descent of man and selection in relation to sex. London: John Murray.
Delibes, M. (1966). USA y yo. Barcelona: Ediciones Destino.

De Waal, F. B. M., \& Harcourt, A. H. (Eds.). (1992). Coalitions and alliances in humans and other animals. Oxford, UK: Oxford University Press.

Durkheim, E. (1951). Suicide. New York: Free Press. (Original work published in 1897).

Fierro, A. (1980). A note on death and dying. Hispanic Journal of Behavioral Sciences, 2(4), 401-406.

Fiske, A. P., Kitayama, S., Markus, H. R., \& Nisbett, R. E. (1998). The cultural matrix of social psychology. In D. T. Gilbert \& S. T. Fiske (Eds.), The handbook of social psychology (Vol. 2, 4th ed., pp. 915-981). New York: McGraw-Hill.

Gould, S. J., \& Vrba, E. S. (1982). Exaptation: A missing term in the science of form. Paleobiology, 8 , 4-15.

Greenberg, J., Pyszczynski, T., \& Solomon, S. (1986). The causes and consequences of a need for self-esteem: A terror management theory. In R. F. Baumeister (Ed.), Public and private self (pp. 189-212). New York: Springer-Verlag.

Greenberg, J., Pyszczynski, T., Solomon, S., Rosenblatt, A., Veeder, M., Kirkland, S. L., et al. (1990). Evidence for terror management theory II: The effects of mortality salience on reactions to those who threaten or bolster the cultural worldview. Journal of Personality and Social Psychology, 58, 308-318.

Greenberg, J., Pyszczynski, T., Solomon, S., Simon, L., \& Breus, M. (1994). Role of consciousness and accessibility of death-related thoughts in mortality salience effects. Journal of Personality and Social Psychology, 67(4), 627-637.

Greenberg, J., Simon, L., Harmon-Jones, E., Solomon, S., \& Lyon, D. (1995). Testing alternative explanations for mortality salience effects: Terror management, value accessibility, or worrisome thoughts? European Journal of Social Psychology, 12(4), 417-433.

Greenberg, J., Solomon, S., \& Pyszczynski, T. (1997). Terror management theory of self-esteem and cultural worldviews: Empirical assessments and conceptual refinements. Advances in Experimental Social Psychology, 29, 61-139.

Hardin, C. D., \& Conley, T. D. (2001). A relational approach to cognition: Shared experience and relationship affirmation in social cognition. In G. B. Moskowitz (Ed.), Cognitive social psychology: The Princeton symposium on the legacy and future of social cognition (pp. 3-17). Mahwah, NJ: Erlbaum.

Hardin, C. D., \& Higgins, E. T. (1996). Shared reality: How social verification makes the 
subjective objective. In R. M. Sorrentino \& E. T. Higgins (Eds.), Handbook of motivation and cognition: Vol. 3. The interpersonal context (pp. 28-84). New York, Guilford.

Hofstede, G. (1991). Culture and organizations. London: McGraw-Hill.

Huber, P. (1981). Robust statistics. New York: Wiley.

Kiev, A. (1976). Cultural perspectives on the range of human behavior. Mental Health and Society, 3(Suppl. 2), 53-56.

Leary, M. R., \& Schreindorfer, L. S. (1997). Unresolved issues with terror management theory. Psychological Inquiry, 8, 26-29.

Levy, R. I. (1973). Tahitians: Mind and experience in the Society Islands. Chicago: University of Chicago Press.

McElreath, R., Boyd, R., \& Richerson, P. J. (2003). Shared norms lead to the evolution of ethnic markers. Current Anthropology, 44, 122-129.

McGregor, I., Zanna, M. P., Holmes, J. G., \& Spencer, S. J. (2001). Compensatory conviction in the face of personal uncertainty: Going to extremes and being oneself. Journal of Personality and Social Psychology, 80, 472-488.

Meléndez, C. (1991). Historia de Costa Rica. San Jose, Costa Rica: EUNED.

Milgram, S. (1974). Obedience to authority: An experimental view. New York: Harper \& Row.

Navarrete, C. D., Kurzban, R., Fessler, D. M. T., \& Kirkpatrick, L. A. (2004). Anxiety and intergroup bias: Terror management or coalitional psychology? Group Processes $\mathcal{E}$ Intergroup Relations, 7, 370-397.

Pyszczynski, T., Greenberg, J., \& Solomon, S. (1997). Why do we need what we need? A terror management perspective on the roots of human social motivation. Psychological Inquiry, 8, 1-20.

Pyszczynski, T., Solomon, S., \& Greenberg, J. (2002). In the wake of 9/11: The psychology of terror. New York: American Psychological Association.

Realo, A., Allik, J., \& Vadi, M. (1997). The hierarchical structure of collectivism. Journal of Research in Personality, 31, 93-116.

Schaller, M., \& Conway, L. G., III. (1999). Influence of impression-management goals on the emerging contents of group stereotypes: Support for a social-evolutionary process. Personality and Social Psychology Bulletin, 25, 819-833.

Schaller, M., Park, J. H., \& Mueller, A. (2003). Fear of the dark: Interactive effects of beliefs about danger and ambient darkness on ethnic stereotypes. Personality and Social Psychology Bulletin, 29, 637-649.

Siefken, S. (1993). The Hispanic perspective on death and dying: A combination of respect, empathy, and spirituality. Pride Institute Journal of Long Term Home Health Care, 12(2), 26-28.

Tooby, J., \& Cosmides, L. (1996). Friendship and the banker's paradox: Other pathways to the evolution of adaptations for altruism. In W. G. Runciman \& J. M. Smith (Eds.), Evolution of social behaviour patterns in primates and man. Proceedings of The British Academy (Vol. 88, pp. 119-143).

Triandis, H. (1995). Individualism and collectivism. Boulder, CO: Westview.

Triandis, H. C., Marin, G., Lisansky, J., \& Betancourt, H. (1984). Simpatia as a cultural script of Hispanics. Journal of Personality and Social Psychology, 47, 1363-1375.

Watson, D., \& Clark, L. A. (1992). Affects separable and inseparable: On the hierarchical arrangement of the negative affects. Journal of Personality and Social Psychology, 62, 489-505.

Watson, D., Clark, L. A., \& Tellegen, A. (1988). Development and validation of brief measures of positive and negative affect: The PANAS scales. Journal of Personality and Social Psychology, 54, 1063-1070.

Paper received 21 March 2004; revised version accepted 14 April 2005.

\section{Biographical note}

CARLOS DAVID NAVARRETE is a NSF Postdoctoral Fellow in Social Psychology at the University of California, Los Angeles. His research on the psychology of intergroup attitudes touches on the intercepts of cultural anthropology, social psychology and evolutionary biology. He is a member of both the Working Group in Experimental Biological Anthropology at the UCLA Center for Behavior, Evolution and Culture, and the Intergroup Relations Interest Group in the Psychology Department at UCLA. Dr. Navarrete holds a Ph.D. in Anthropology from UCLA and has conducted fieldwork in rural Costa Rica and in urban Los Angeles. 